\title{
Common Fixed Point Theorems In Complex Valued Metric Spaces For Weakly Compatible Mappings, E.A. Property And CLR Property
}

\author{
R K Gujetiya ${ }^{1}$, Dheeraj Kumari Mali ${ }^{2, *}$, Mala Hakwadiya ${ }^{3}$ \\ ${ }^{I}$ Associate Professor \& Head, Department of Mathematics, Govt. P. G. College, Neemuch, India \\ ${ }^{2}$ Research Scholar, Pacific Academy Of Higher Education and Research University Udaipur, Rajasthan, India \\ ${ }^{3}$ Research Scholar, Pacific Academy Of Higher Education and Research University Udaipur, Rajasthan, India
}

Abstract: In this paper, we prove a common fixed point theorem in complex valued metric space for weakly compatible mappings. Also, we prove common fixed point theorems for weakly compatible mappings with E.A. property and CLR property. We will generalized and extended the result of S.M. Kang [7].

AMS Subject Classification: $47 H 10,54 H 25$

Key Words: CLR property, complex valued metric space, E.A. property, weakly compatible mapping.

\section{Introduction}

In 2011, Azam et al. [1] introduced the notion of complex valued metric space which is a generalization of the classical metric space and established some fixed point results for mappings satisfying a rational inequality. Jungck [3] and Vetro [2] introduced the concept of weakly compatible maps. In 2002, Aamri and Moutawakil [4] introduced the notion of E.A. property. In 2011, Sintunavarat and Kumam [8] introduced the notion of CLR property. In 2013, Verma and Pathak [5] defined the 'max' function for partial order relation $\precsim$ A complex number $\mathrm{z} \in \mathrm{C}$ is an ordered pair of real numbers, whose first coordinate is called $\operatorname{Re}(\mathrm{z})$ and second coordinate is called $\operatorname{Im}(\mathrm{z})$.

\section{Preliminaries}

Definition 2.1.[7] Let $X$ be a nonempty set. Suppose that the mapping $d: X \times X \rightarrow \mathbb{C}$ satisfies

(1) $0 \lesssim d(x, y)$ for all $x, y \in X$ and $d(x, y)=0$ if and only if $x=y$;

(2) $\mathrm{d}(\mathrm{x}, \mathrm{y})=\mathrm{d}(\mathrm{y}, \mathrm{x})$ for all $\mathrm{x}, \mathrm{y} \in \mathrm{X}$;

(3) $\mathrm{d}(\mathrm{x}, \mathrm{y}) \precsim \mathrm{d}(\mathrm{x}, \mathrm{z})+\mathrm{d}(\mathrm{z}, \mathrm{y})$ for all $\mathrm{x}, \mathrm{y}, \mathrm{z} \in \mathrm{X}$.

Then $d$ is called a complex valued metric on $X$ and $(X, d)$ is called a complex valued metric space.

Example 2.2. [7] Let $X=\mathbb{C}$. Define the mapping $d: X \times X \rightarrow \mathbb{C}$ by $d\left(z_{1}, z_{2}\right)=2 i\left|z_{1}-z_{2}\right|$ for all $z_{1}, z_{2} \in X$. Then $(\mathrm{X}, \mathrm{d})$ is a complex valued metric space.

Definition 2.3. [7] Let $(X, d)$ be a complex valued metric space and $\left\{x_{n}\right\}$ be a sequence in $X$.

(1) If for every $\mathrm{c} \in \mathbb{C}$ with $0<\mathrm{c}$, there exists $\mathrm{N} \in \mathbb{N}$ such that $\mathrm{d}\left(\mathrm{x}_{\mathrm{n}}, \mathrm{x}\right)<\mathrm{c}$ for all $\mathrm{n} \geq \mathrm{N}$ then $\left\{\mathrm{x}_{\mathrm{n}}\right\}$ is said to be convergent to $\mathrm{x} \in \mathrm{X}$, and we denote this by $\mathrm{x}_{\mathrm{n}} \rightarrow \mathrm{x}$ as $\mathrm{n} \rightarrow \infty$ or $\lim _{\mathrm{n} \rightarrow \infty} \mathrm{x}_{\mathrm{n}}=\mathrm{x}$.

(2) If for every $c \in \mathbb{C}$ with $0 \prec c$, there exists $N \in \mathbb{N}$ such that $d\left(x_{n}, x_{n+m}\right) \prec c$ for all $n \geq N$, where $m \in \mathbb{N}$, then $\left\{\mathrm{x}_{\mathrm{n}}\right\}$ is said to be Cauchy sequence.

(3) If every Cauchy sequence in $X$ is convergent, then $(X, d)$ is said to be a complete complex valued metric space.

Lemma 2.4. [7] Let $(X, d)$ be a complex valued metric space and $\left\{x_{n}\right\}$ be a sequence in $X$. Then $\left\{x_{n}\right\}$ converges to $\mathrm{x}$ if and only if $\left|\mathrm{d}\left(\mathrm{x}_{\mathrm{n}}, \mathrm{x}\right)\right| \rightarrow 0$ as $\mathrm{n} \rightarrow \infty$.

Lemma 2.5. [7] Let $(X, d)$ be a complex valued metric space and $\left\{x_{n}\right\}$ be a sequence in $X$. Then $\left\{x_{n}\right\}$ is a Cauchy sequence if and only if $\left|d\left(x_{n}, x_{n+m}\right)\right| \rightarrow 0$ as $n \rightarrow \infty$, where $m \in N$.

Definition 2.6. [7] Let $f$ and $g$ be two self-mappings of a metric space (X, d). Then a pair (f, $g$ ) is said to be weakly compatible if they commute at coincidence points.

Definition 2.7. [7] Let $f$ and $g$ be two self-mappings of a metric space (X, d). Then a pair (f,g) is said to satisfy E.A. property if there exists a sequence $\left\{\mathrm{x}_{\mathrm{n}}\right\}$ in $\mathrm{X}$ such that $\lim _{\mathrm{n} \rightarrow \infty} \mathrm{fx}_{\mathrm{n}}=\lim _{\mathrm{n} \rightarrow \infty} \mathrm{gx}_{\mathrm{n}}=\mathrm{t}$ for some $\mathrm{t} \in \mathrm{X}$.

Definition 2.8. [7] Let $f$ and $g$ be two self-mappings of a metric space (X, d). Then a pair (f,g) is said to satisfy $\mathrm{CLR}_{\mathrm{f}}$ property if there exists a sequence $\left\{\mathrm{x}_{\mathrm{n}}\right\}$ in $\mathrm{X}$ such that $\lim _{\mathrm{n} \rightarrow \infty} \mathrm{fx}_{\mathrm{n}}=\lim _{\mathrm{n} \rightarrow \infty} \mathrm{gx}_{\mathrm{n}}=\mathrm{fx}$ for some $\mathrm{x} \in \mathrm{X}$.

Example 2.9. [7] Let $X=\mathbb{C}$. Define the mapping $d: X \times X \rightarrow \mathbb{C}$ by d(z_1,z_2)=2i|z_1-z_2 for all $z_{1}, z_{2} \in$ $X$. Then $(X, d)$ is a complex valued metric space. Define $S$ and $T: X \rightarrow X$ by Sz $=z+\bar{i}$ and $T z=2 z$ for all $\mathrm{z} \in X$, respectively. Consider a sequence $\left\{z_{n}\right\}=\left\{i-\frac{1}{n}\right\}(n \in \mathbb{N})$ in $X$. Then $\lim _{n \rightarrow \infty} S z_{n}=\lim _{n \rightarrow \infty}\left(z_{n}+i\right)=$ 
$2 \mathrm{i}$ and $\lim _{\mathrm{n} \rightarrow \infty} \mathrm{Tz}_{\mathrm{n}}=\lim _{\mathrm{n} \rightarrow \infty} 2 \mathrm{z}_{\mathrm{n}}=2 \mathrm{i}$ where $2 \mathrm{i} \in \mathrm{X}$. Thus, $\mathrm{S}$ and T satisfy E.A. property. Also, we have $\lim _{n \rightarrow \infty} S z_{n}=\lim _{n \rightarrow \infty} T z_{n}=2 i=S i$. where $2 i \in X$. Thus, $S$ and $T$ satisfy CLR property.

Definition 2.10. [7] Define the 'max' function for the partial order relation $\lesssim$ by

(1) $\max \left\{\mathrm{z}_{1}, \mathrm{z}_{2}\right\}=\mathrm{z}_{2}$ if and only ifz $\mathrm{z}_{1} \precsim \mathrm{z}_{2}$.

(2) If $\mathrm{z}_{1} \precsim \max \left\{\mathrm{z}_{2}, \mathrm{z}_{3}\right\}$, then $\mathrm{z}_{1} \precsim \mathrm{z}_{2}$ or $\mathrm{z}_{1} \precsim \mathrm{z}_{3}$.

(3) $\max \left\{z_{1}, z_{2}\right\}=z_{2}$ if and only if $z_{1} \lesssim z_{2}$ or $\left|z_{1}\right| \leq\left|z_{2}\right|$.

Using above Definition, we have the following lemma.

Lemma 2.11. [7] Let $\mathrm{z}_{1}, \mathrm{z}_{2}, \mathrm{z}_{3} \ldots . . \in \mathbb{C}$ and the partial order relation $\lesssim$ is defined on $\mathbb{C}$. Then following statements are easy to prove.

(i) If $\mathrm{z}_{1} \lesssim \max \left\{\mathrm{z}_{2}, \mathrm{z}_{3}\right\}$, then $\mathrm{z}_{1} \lesssim \mathrm{z}_{2}$ if $\mathrm{z}_{3} \lesssim \mathrm{z}_{2}$;

(ii) If $\mathrm{z}_{1} \precsim \max \left\{\mathrm{z}_{2}, \mathrm{z}_{3}, \mathrm{z}_{4}\right\}$, then $\mathrm{z}_{1} \lesssim \mathrm{z}_{2}$ if $\max \left\{\mathrm{z}_{3}, \mathrm{z}_{4}\right\} \precsim \mathrm{z}_{2}$;

(iii) If $\mathrm{z}_{1} \precsim \max \left\{\mathrm{z}_{2}, \mathrm{z}_{3}, \mathrm{z}_{4}, \mathrm{z}_{5}\right\}$, then $\mathrm{z}_{1} \precsim \mathrm{z}_{2}$ if $\max \left\{\mathrm{z}_{3}, \mathrm{z}_{4}, \mathrm{z}_{5}\right\} \lesssim \mathrm{z}_{2}$, and so on.

\section{Main Result}

Theorem 3.1 : Let A, B, D, M, S and T be six self mappings of a complex valued metric space (X, d) satisfying: 1. $\mathrm{S}(\mathrm{X}) \subset \mathrm{BD}(\mathrm{X})$ and $\mathrm{T}(\mathrm{X}) \subset \mathrm{AM}(\mathrm{X})$

2. For each $\mathrm{x}, \mathrm{y} \in \mathrm{X}$, there exists $\alpha, \beta, \gamma$ and $\eta$ are non negative real number with $\alpha+\beta+\gamma+\eta<1$, such that $\mathrm{d}($ Sx, Ty $) \precsim \alpha\left[\mathrm{d}\left(\right.\right.$ BDy, Ty) $\left.\frac{1+\mathrm{d}(\mathrm{AMx}, \mathrm{Sx})}{1+\mathrm{d}(\mathrm{AMx}, \mathrm{BDy})}\right]+\beta[\max \{\mathrm{d}(\mathrm{AMx}, \mathrm{By}), \mathrm{d}(\mathrm{AMx}, \mathrm{Sx}), \mathrm{d}($ BDy, Ty $)\}]$

$$
+\gamma[d(T y, S x)]+\eta\left[\frac{d(T y, B D y) d(A M x, S x)}{d(T y, A M x)+d(S x, B D y)+d(T y, S x)}\right]
$$

3. The pair (AM, S) and (BD, T) are weakly compatible.

4. Suppose that One of $A(X), B(X), S(X)$ and $T(X)$ is complete subspace of $X$.

5. The pair (AM, S) and (BD, T) are commute.

Then A, B, D, M, S and T have a unique common fixed point.

Proof: Let $x_{0} \in X$. Since $\mathrm{S}(\mathrm{X}) \subset \mathrm{BD}(\mathrm{X})$ and $\mathrm{T}(\mathrm{X}) \subset \mathrm{AM}(\mathrm{X})$, define for each $\mathrm{n} \geq 0$, the sequence $\left\{y_{n}\right\}$ in $\mathrm{X}$ by

$$
y_{2 n+1}=S x_{2 n}=B D x_{2 n+1} \text { and } y_{2 n+2}=T x_{2 n+1}=A M x_{2 n+2}
$$

Case I : Suppose that $y_{2 n}=y_{2 n+1}$ for some $n$. Then by (2), we have $y_{2 n+2}=y_{2 n+1}$, and so, $y_{m}=y_{2 n}$ for every $\mathrm{m}>2 \mathrm{n}$. Thus, the sequence $\left\{y_{n}\right\}$ is a Cauchy sequence. The same conclusion holds if $y_{2 n+1}=y_{2 n+2}$ for some $n$.

Case II : Assume that $y_{n} \neq y_{n+1}$ for all n. Putting $x=x_{2 n}$ and $y=x_{2 n-1}$ in (2), we have

$$
\begin{array}{r}
d\left(S x_{2 n}, T x_{2 n-1}\right) \lesssim \alpha\left[d\left(B D x_{2 n-1}, T x_{2 n-1}\right) \frac{1+d\left(A M x_{2 n}, S x_{2 n}\right)}{1+d\left(A M x_{2 n}, B D x_{2 n-1}\right)}\right] \\
+\beta\left[\max \left\{d\left(A M x_{2 n}, B D x_{2 n-1}\right), d\left(A M x_{2 n}, S x_{2 n}\right), d\left(B D x_{2 n-1}, T x_{2 n-1}\right)\right\}\right]+ \\
\gamma\left[d\left(T x_{2 n-1}, S x_{2 n}\right)\right]+\eta\left[\frac{d\left(T x_{2 n-1}, B D x_{2 n-1}\right) d\left(A M x_{2 n}, S x_{2 n}\right)}{d\left(T x_{2 n-1}, A M x_{2 n}\right)+d\left(S x_{2 n}, B D x_{2 n-1}\right)+d\left(T x_{2 n-1}, S x_{2 n}\right)}\right]
\end{array}
$$

$d\left(y_{2 n+1}, y_{2 n}\right)$

$$
\begin{array}{r}
\lesssim \alpha\left[d\left(y_{2 n-1}, y_{2 n}\right) \frac{1+d\left(y_{2 n}, y_{2 n+1}\right)}{1+d\left(y_{2 n}, y_{2 n-1}\right)}\right]+\beta\left[\max \left\{d\left(y_{2 n}, y_{2 n-1}\right), d\left(y_{2 n}, y_{2 n+1}\right), d\left(y_{2 n-1}, y_{2 n}\right)\right\}\right]+ \\
\gamma\left[d\left(y_{2 n}, y_{2 n+1}\right)\right]+\eta\left[\frac{d\left(y_{2 n}, y_{2 n-1}\right) d\left(y_{2 n}, y_{2 n+1}\right)}{d\left(y_{2 n}, y_{2 n}\right)+d\left(y_{2 n+1}, y_{2 n-1}\right)+d\left(y_{2 n}, y_{2 n+1}\right)}\right] \\
d\left(y_{2 n+1}, y_{2 n}\right) \lesssim \alpha d\left(y_{2 n}, y_{2 n+1}\right)+\beta d\left(y_{2 n}, y_{2 n+1}\right)+\gamma d\left(y_{2 n}, y_{2 n+1}\right)+\eta d\left(y_{2 n}, y_{2 n+1}\right)
\end{array}
$$

Thus, we have $\left|d\left(y_{2 n}, y_{2 n+1}\right)\right| \leq(\alpha+\beta+\gamma+\eta)\left|d\left(y_{2 n}, y_{2 n+1}\right)\right|$

Which is a contradiction to $(\alpha+\beta+\gamma+\eta)<1$. Conversely we have

$$
d\left(y_{2 n+1}, y_{2 n}\right) \lesssim d\left(y_{2 n}, y_{2 n-1}\right)
$$

Thus, we have $d\left(y_{2 n+1}, y_{2 n}\right) \precsim(\alpha+\beta+\gamma+\eta) d\left(y_{2 n}, y_{2 n-1}\right)$

On putting $x=x_{2 n-2}$ and $y=x_{2 n-1}$ in (2), we have

$$
\begin{aligned}
& d\left(S x_{2 n-2}, T x_{2 n-1}\right) \lesssim \alpha\left[d\left(B D x_{2 n-1}, T x_{2 n-1}\right) \frac{1+d\left(A M x_{2 n-2}, S x_{2 n-2}\right)}{1+d\left(A M x_{2 n-2}, B D x_{2 n-1}\right)}\right] \\
& +\beta\left[\max \left\{d\left(A M x_{2 n-2}, B D x_{2 n-1}\right), d\left(A M x_{2 n-2}, S x_{2 n-2}\right), d\left(B D x_{2 n-1}, T x_{2 n-1}\right)\right\}\right]+ \\
& \gamma\left[d\left(T x_{2 n-1}, S x_{2 n-2}\right)\right]+\eta\left[\frac{d\left(T x_{2 n-1}, B D x_{2 n-1}\right) d\left(A M x_{2 n-2}, S x_{2 n-2}\right)}{d\left(T x_{2 n-1}, A M x_{2 n-2}\right)+d\left(S x_{2 n-2}, B D x_{2 n-1}\right)+d\left(T x_{2 n-1}, S x_{2 n-2}\right)}\right] \\
& d\left(y_{2 n-1}, y_{2 n}\right) \lesssim \alpha\left[d\left(y_{2 n-1}, y_{2 n}\right) \frac{1+d\left(y_{2 n-2}, y_{2 n-1}\right)}{1+d\left(y_{2 n-2}, y_{2 n-1}\right)}\right] \\
& +\beta\left[\max \left\{d\left(y_{2 n-2}, y_{2 n-1}\right), d\left(y_{2 n-2}, y_{2 n-1}\right), d\left(y_{2 n-1}, y_{2 n}\right)\right\}\right]+\gamma\left[d\left(y_{2 n}, y_{2 n-1}\right)\right]
\end{aligned}
$$




$$
+\eta\left[\frac{d\left(y_{2 n}, y_{2 n-1}\right) d\left(y_{2 n-2}, y_{2 n-1}\right)}{d\left(y_{2 n}, y_{2 n-2}\right)+d\left(y_{2 n-1}, y_{2 n-1}\right)+d\left(y_{2 n}, y_{2 n-1}\right)}\right]
$$

$d\left(y_{2 n-1}, y_{2 n}\right) \precsim \alpha d\left(y_{2 n-1}, y_{2 n}\right)+\beta d\left(y_{2 n-1}, y_{2 n}\right)+\gamma d\left(y_{2 n-1}, y_{2 n}\right)+\eta d\left(y_{2 n-1}, y_{2 n}\right)$

$(1-\alpha) d\left(y_{2 n-1}, y_{2 n}\right) \precsim(\beta+\gamma+\eta) d\left(y_{2 n-1}, y_{2 n}\right)$

Thus, we have $\quad\left|d\left(y_{2 n-1}, y_{2 n}\right)\right| \leq\left(\frac{\beta+\gamma+\eta}{1-\alpha}\right)\left|d\left(y_{2 n-1}, y_{2 n}\right)\right|$

Which is a contradiction to $(\beta+\gamma+\eta)<1$. Then we have

$$
\left|d\left(y_{2 n-1}, y_{2 n}\right)\right| \leq\left(\frac{\beta+\gamma+\eta}{1-\alpha}\right)\left|d\left(y_{2 n-2}, y_{2 n-1}\right)\right|
$$

Define $k=\max \left[(\alpha+\beta+\gamma+\eta),\left(\frac{\beta+\gamma+\eta}{1-\alpha}\right)\right]$ conversely, it can be concluded that

$d\left(y_{n}, y_{n+1}\right) \lesssim k d\left(y_{n-1}, y_{n}\right)$

$d\left(y_{n}, y_{n+1}\right) \lesssim k^{2} d\left(y_{n-2}, y_{n-1}\right) \lesssim \ldots \ldots \ldots \ldots . . . . k^{n} d\left(y_{0}, y_{1}\right)$

Now for all $\mathrm{m}>\mathrm{n}$, we have

$$
\begin{aligned}
& d\left(y_{m}, y_{n}\right) \precsim d\left(y_{n}, y_{n+1}\right)+d\left(y_{n+1}, y_{n+2}\right)+\cdots \ldots+d\left(y_{m-1}, y_{m}\right) \\
& d\left(y_{m}, y_{n}\right) \precsim k^{n} d\left(y_{0}, y_{1}\right)+k^{n+1} d\left(y_{0}, y_{1}\right)+\cdots+k^{m-1} d\left(y_{0}, y_{1}\right)
\end{aligned}
$$

Therefore we have,

$$
\left|d\left(y_{m}, y_{n}\right)\right| \leq \frac{k^{n}}{1-k}\left|d\left(y_{0}, y_{1}\right)\right|
$$

Hence, we obtain $\lim _{n \rightarrow \infty}\left|d\left(y_{m}, y_{n}\right)\right|=0$, hence $\left\{y_{n}\right\}$ is a Cauchy sequence.

Case III : Suppose that $\operatorname{AM}(X)$ is complete then the sequence $\left\{y_{2 n}\right\}$ is contained in $\operatorname{AM}(X)$ and has a limit in $\mathrm{AM}(\mathrm{X})$, say $\mathrm{u}$, that is $\lim _{n \rightarrow \infty} y_{2 n}=u$. Since $\mathrm{u} \in \mathrm{AM}(\mathrm{X})$, there exists $\mathrm{v} \in \mathrm{X}$ such that $A M v=u$.

Now, we shall prove $S v=u$. Let $S v \neq u$. From (2) putting $x=v$ and $y=x_{2 n-1}$, we have

$$
\begin{gathered}
d\left(S v, T x_{2 n-1}\right) \lesssim \alpha\left[d\left(B D x_{2 n-1}, T x_{2 n-1}\right) \frac{1+d(A M v, S v)}{1+d\left(A M v, B D x_{2 n-1}\right)}\right] \\
+\beta\left[\operatorname { m a x } \left[\frac{\left.\left.d\left(A M v, B D x_{2 n-1}\right), d(A M v, S v), d\left(B D x_{2 n-1}, T x_{2 n-1}\right)\right\}\right]+\gamma\left[d\left(T x_{2 n-1}, S v\right)\right]+}{\eta\left[\frac{d\left(T x_{2 n-1}, B D x_{2 n-1}\right) d(A M v, S v)}{d\left(T x_{2 n-1}, A M v\right)+d\left(S v, B D x_{2 n-1}\right)+d\left(T x_{2 n-1}, S v\right)}\right]}\right.\right. \\
\lesssim \alpha\left[d\left(y_{2 n-1}, y_{2 n}\right) \frac{1+d(u, S v)}{1+d\left(u, y_{2 n-1}\right)}\right]+\beta\left[\max \left\{d\left(u, y_{2 n-1}\right), d(u, S v), d\left(y_{2 n-1}, y_{2 n}\right)\right\}\right]+\gamma\left[d\left(y_{2 n}, S v\right)\right] \\
+\eta\left[\frac{d\left(y_{2 n-1}, y_{2 n}\right) d(S v, u)}{d\left(y_{2 n}, u\right)+d\left(S v, y_{2 n-1}\right)+d\left(y_{2 n}, S v\right)}\right]
\end{gathered}
$$

As the sequence $\left\{y_{2 n-1}\right\}$ is convergent to $u$, therefore

Thus letting $\mathrm{n} \rightarrow \infty$, we have

$$
\lim _{n \rightarrow \infty} d\left(u, y_{2 n-1}\right)=\lim _{n \rightarrow \infty} d\left(y_{2 n}, y_{2 n-1}\right)=0
$$

$\lesssim \alpha\left[d(u, u) \frac{1+d(u, S v)}{1+d(u, u)}\right]+\beta[\max \{d(u, u), d(u, S v), d(u, u)\}]+\gamma[d(u, S v)]$

$$
+\eta\left[\frac{d(u, u) d(S v, u)}{d(u, u)+d(S v, u)+d(u, S v)}\right]
$$

$\precsim \beta d(u, S v)+\gamma d(u, S v)$

$d(S v, u) \lesssim(\beta+\gamma) d(u, S v)$

That is, $\quad|d(S v, u)| \leq(\beta+\gamma)|d(S v, u)|$

Which is a contradiction to $(\beta+\gamma)<1$. Hence $u=S v=A M v$. Now, since $S(X) \subset B D(X), S u=u \in$ $B D(X)$. There exists $\mathrm{w} \in \mathrm{X}$ such that $B D w=u$. By using the same argument as above, one can easily verify that $T w=u=B D w$, that is, $\mathrm{w}$ is the coincidence point of the pair $(\mathrm{BD}, \mathrm{T})$.

The same result hold if we assume that $\mathrm{BD}(\mathrm{X})$ is complete.

Now if $\mathrm{T}(\mathrm{X})$ is complete, then by (2.1), $u \in T(X) \subset A M(X)$. Similarly, if $\mathrm{S}(\mathrm{X})$ is complete, then $u \in S(X)$ $\subset \mathrm{BD}(\mathrm{X})$.

Now, since the pair (AM, S) and (BD, T) are weakly compatible, so $u=S v=A M v=T w=B D w$ and hence they commute at their coincidence point that is, $A M u=A M(S v)=S(A M v)=S u$ and $B D u=$ $B D(T w)=T(B D w)=T u$.

Now, we claim that $T u=u$. Let $T u \neq u$. From(2), we have

$$
\begin{array}{r}
d(S v, T u) \lesssim \alpha\left[d(B D u, T u) \frac{1+d(A M v, S v)}{1+d(A M v, B D u)}\right]+\beta[\max \{d(A M v, B D u), d(A M v, S v), d(B D u, T u)\}]+ \\
\gamma[d(T u, S v)]+\eta\left[\frac{d(T u, B D u) d(A M v, S v)}{d(T u, A M v)+d(S v, B D u)+d(T u, S v)}\right]
\end{array}
$$




$$
d(u, T u) \lesssim \alpha\left[d(T u, T u) \frac{1+d(u, u)}{1+d(u, T u)}\right]+\beta[\max \{d(u, T u), d(u, u), d(T u, T u)\}]+\gamma[d(T u, u)]
$$

Thus,

$$
d(u, T u) \lesssim \beta d(u, T u)+\gamma d(u, T u)
$$

$$
+\eta\left[\frac{d(T u, T u) d(u, u)}{d(T u, u)+d(u, T u)+d(T u, u)}\right]
$$

That is,

$$
|d(u, T u)| \leq(\beta+\gamma)|d(u, T u)|
$$

Which is a contradiction to $(\beta+\gamma)<1$. Therefore $T u=u$. Since $B D u=T u$, which implies that $B D u=$ $u$. Similarly we can prove that $S u=u$. Since $u=S u$, which implies that $A M u=u=S u$.

Now to prove $M u=u$, using (2), putting $x=M u$ and $y=u$, we have

$$
\begin{array}{r}
d(S(M u), T u) \lesssim \alpha\left[d(B D u, T u) \frac{1+d(A M(M u), S(M u))}{1+d(A M(M u), B D u)}\right] \\
+\beta[\max \{d(A M(M u), B D u), d(A M(M u), S(M u)), d(B D u, T u)\}]+\gamma[d(T u, S(M u))] \\
+\eta\left[\frac{d(T u, B D u) d(A M(M u), S(M u))}{d(T u, A M(M u))+d(S(M u), B D u)+d(T u, S(M u))}\right]
\end{array}
$$

$d(M u, u) \precsim \beta d(M u, u)+\gamma d(M u, u)$

That is, $\quad|d(u, M u)| \leq(\beta+\gamma)|d(u, M u)|$

Which is a contradiction to $(\beta+\gamma)<1$. Therefore $M u=u$. Since $A M u=u$ which implies that $A u=u$. Now, to prove $D u=u$, using(2), putting $x=u, y=D u$, we have

$$
\begin{aligned}
& d(S u, T(D u)) \lesssim \alpha\left[d(B D(D u), T(D u)) \frac{1+d(A M u, S u)}{1+d(A M u, B D(D u))}\right] \\
& +\beta[\max \{d(A M u, B D(D u)), d(A M u, S u), d(B D(D u), T(D u))\}]+\gamma[d(T(D u), S u)] \\
& +\eta\left[\frac{d(T(D u), B D(D u)) d(A M u, S u)}{d(T(D u), A M u)+d(S u, B D(D u))+d(T(D u), S u)}\right]
\end{aligned}
$$

$d(u, D u) \lesssim \alpha\left[d(D u, D u) \frac{1+d(u, u)}{1+d(u, D u)}\right]+\beta[\max \{d(u, D u), d(u, u), d(D u, D u)\}]+\gamma[d(D u, u)]$

$$
+\eta\left[\frac{d(D u, D u) d(u, u)}{d(D u, u)+d(u, D u)+d(D u, u)}\right]
$$

$d(D u, u) \lesssim \beta d(D u, u)+\gamma d(D u, u)$

That is, $\quad|d(u, D u)| \leq(\beta+\gamma)|d(u, D u)|$

Which is a contradiction to $(\beta+\gamma)<1$. Therefore $D u=u$. Since $B D u=u$ which implies that $B u=u$. Thus combining all the above result, we have $A u=B u=D u=M u=S u=T u=u$. Hence $u$ is a common fixed point of A, B, D, M, S and T.

Uniqueness: Let $z(z \neq u)$ be an another common fixed point of A, B, D, M, S and T. Then from (2), we have

$$
\begin{aligned}
& d(u, z)=d(S u, T z) \lesssim \alpha\left[d(B D z, T z) \frac{1+d(A M u, S u)}{1+d(A M u, B D z)}\right] \\
& +\beta[\max \{d(A M u, B D z), d(A M u, S u), d(B D z, T z)\}]+\gamma[d(T z, S u)] \\
& +\eta\left[\frac{d(T z, B D z) d(A M u, S u)}{d(T z, A M u)+d(S u, B D z)+d(T z, S u)}\right]
\end{aligned}
$$

$d(u, z) \lesssim \alpha\left[d(z, z) \frac{1+d(u, u)}{1+d(u, z)}\right]+\beta[\max \{d(u, z), d(u, u), d(z, z)\}]+\gamma[d(z, u)]$

$$
+\eta\left[\frac{d(z, z) d(u, u)}{d(z, u)+d(u, z)+d(z, u)}\right]
$$

$d(u, z) \lesssim \beta d(u, z)+\gamma d(u, z)$

That is, $\quad|d(u, z)| \leq(\beta+\gamma)|d(u, z)|$

Which is a contradiction to $(\beta+\gamma)<1$. Therefore $\mathrm{z}=u$. Hence $\mathrm{u}$ is a unique common fixed point of $\mathrm{A}, \mathrm{B}$,

$\mathrm{D}, \mathrm{M}, \mathrm{S}$ and $\mathrm{T}$.

Corollary : Let A, B, S and T be self mappings of a complex valued metric space $(X, d)$ satisfying:

1. $S(X) \subset B(X)$ and $T(X) \subset A(X)$

2. For each $\mathrm{x}, \mathrm{y} \in \mathrm{X}$, there exists $\alpha, \beta, \gamma$ and $\eta$ are non negative real numbers with $\alpha+\beta+\gamma+\eta<1$, such that 
$d(S x, T y) \lesssim \alpha\left[d(B y, T y) \frac{1+d(A x, S x)}{1+d(A x, B y)}\right]+\beta[\max \{d(A x, B y), d(A x, S x), d(B y, T y)\}]$

3. The pair (A, S) and (B, T) are weakly compatible.

$$
+\gamma[d(T y, S x)]+\eta\left[\frac{d(T y, B y) d(A x, S x)}{d(T y, A x)+d(S x, B y)+d(T y, S x)}\right]
$$

4. One of $A(X), B(X), S(X)$ and $T(X)$ is complete.

Then $\mathrm{A}, \mathrm{B}, \mathrm{S}$ and $\mathrm{T}$ have a unique common fixed point.

Fixed Point Theorem For Weakly Compatible Mappings With E.A. Property

Theorem 3.2 : Let A, B, D, M, S and T be self mappings of a complex valued metric space (X, d) satisfying: 1. $S(X) \subset B D(X)$ and $T(X) \subset A M(X)$

2. For each $\mathrm{x}, \mathrm{y} \in \mathrm{X}$, there exists $\alpha, \beta, \gamma$ and $\eta$ are non negative real numbers with $\alpha+\beta+\gamma+\eta<1$, such that $d(S x, T y) \precsim \alpha\left[d(B D y, T y) \frac{1+d(A M x, S x)}{1+d(A M x, B D y)}\right]+$

$$
\begin{array}{r}
\beta[\max [\operatorname{sid}(A M x, B D y), d(A M x, S x), d(B D y, T y)\}]+\gamma[d(T y, S x)]+ \\
\eta\left[\frac{d(T y, B D y) d(A M x, S x)}{d(T y, A M x)+d(S x, B D y)+d(T y, S x)}\right]
\end{array}
$$

3. The pair (AM, S) and (BD, T) are weakly compatible.

4. The pair (AM, S) and (BD, T) satisfy the E.A. property.

5. One of the $\mathrm{AM}(\mathrm{X}), \mathrm{BD}(\mathrm{X}), \mathrm{S}(\mathrm{X})$ and $\mathrm{T}(\mathrm{X})$ is closed subspace of $\mathrm{X}$.

6. The pair (AM, S) and (BD, T) are commute.

Then A, B, D, M, S and T have a unique common fixed point.

Proof: Suppose that(AM, S) satisfies the E.A. property. Then there exists a sequence $\left\{x_{n}\right\}$ in $X$ such that $A M x_{n}=S x_{n}=z$ for some $\mathrm{z} \in \mathrm{X}$. Since $\mathrm{S}(\mathrm{X}) \subset \mathrm{BD}(\mathrm{X})$, there exists a sequence $\left\{y_{n}\right\}$ in $\mathrm{X}$ such that $S x_{n}=$ $B D y_{n}=z$. Hence $\lim _{n \rightarrow \infty} B D y_{n}=z$.

We shall show that $\lim _{n \rightarrow \infty} T y_{n}=z$. Let $\lim _{n \rightarrow \infty} T y_{n}=t \neq z$. From (2), putting $x=x_{n}$ and $y=y_{n}$, we have $d\left(S x_{n}, T y_{n}\right) \lesssim \alpha\left[d\left(B D y_{n}, T y_{n}\right) \frac{1+d\left(A M x_{n}, S x_{n}\right)}{1+d\left(A M x_{n}, B D y_{n}\right)}\right]$

$+\beta\left[\max \left\{d\left(A M x_{n}, B D y_{n}\right), d\left(A M x_{n}, S x_{n}\right), d\left(B D y_{n}, T y_{n}\right)\right\}\right]+\gamma\left[d\left(T y_{n}, S x_{n}\right)\right]$ $+\eta\left[\frac{d\left(T y_{n}, B D y_{n}\right) d\left(A M x_{n}, S x_{n}\right)}{d\left(T y_{n}, A M x_{n}\right)+d\left(S x_{n}, B D y_{n}\right)+d\left(T y_{n}, S x_{n}\right)}\right]$

Letting $\mathrm{n} \rightarrow \infty$, we have

$d(z, t) \lesssim \alpha\left[d(z, t) \frac{1+d(z, z)}{1+d(z, z)}\right]+\beta[\max \{d(z, z), d(z, z), d(z, t)\}]+\gamma[d(t, z)]$

$$
+\eta\left[\frac{d(t, z) d(z, z)}{d(t, z)+d(z, z)+d(t, z)}\right]
$$

$d(z, t) \lesssim \alpha[d(z, t)]+\beta[d(z, t)]+\gamma[d(t, z)]$

That is, $\quad|d(z, t)| \leq(\alpha+\beta+\gamma)|d(z, t)|$

Which is a contradiction to $(\alpha+\beta+\gamma)<1$. Therefore $t=z$, that is $\lim _{n \rightarrow \infty} T y_{n}=z$.

Suppose that $\mathrm{BD}(\mathrm{X})$ is a closed spaces of $\mathrm{X}$. Then there exists $\mathrm{u} \in \mathrm{X}$ such that $z=B D u$. Subsequently, we have

$$
\lim _{n \rightarrow \infty} T y_{n}=\lim _{n \rightarrow \infty} S x_{n}=\lim _{n \rightarrow \infty} A M x_{n}=\lim _{n \rightarrow \infty} B D y_{n}=z=B D u
$$

Now, we prove that $T u=z$. From (2), putting $\mathrm{x}=x_{n}$ and $\mathrm{y}=\mathrm{u}$, we have

$$
\begin{array}{r}
d\left(S x_{n}, T u\right) \lesssim \alpha\left[d(B D u, T u) \frac{1+d\left(A M x_{n}, S x_{n}\right)}{1+d\left(A M x_{n}, B D u\right)}\right] \\
+\beta\left[\max \left\{d\left(A M x_{n}, B D u\right), d\left(A M x_{n}, S x_{n}\right), d(B D u, T u)\right\}\right]+\gamma\left[d\left(T u, S x_{n}\right)\right] \\
+\eta\left[\frac{d(T u, B D u) d\left(A M x_{n}, S x_{n}\right)}{d\left(T u, A M x_{n}\right)+d\left(S x_{n}, B D u\right)+d\left(T u, S x_{n}\right)}\right]
\end{array}
$$

Letting $\mathrm{n} \rightarrow \infty$, we have

$$
\begin{aligned}
d(z, T u) \lesssim \alpha\left[d(z, T u) \frac{1+d(z, z)}{1+d(z, z)}\right]+\beta[\max \{d(z, z), d(z, z), d(z, T u)\}] & \\
& +\gamma[d(T u, z)]+\eta\left[\frac{d(T u, z) d(z, z)}{d(T u, z)+d(z, z)+d(T u, z)}\right]
\end{aligned}
$$

$d(z, T u) \precsim \alpha[d(z, T u)]+\beta[d(z, T u)]+\gamma[d(T u, z)]$

That is, $\quad|d(z, T u)| \leq(\alpha+\beta+\gamma)|d(z, T u)|$ 
Which is a contradiction to $(\alpha+\beta+\gamma)<1$. Therefore $T u=z=B D u$. Now, since $\mathrm{T}(\mathrm{X}) \subset \mathrm{AM}(\mathrm{X}), T u=$ $z \in A M(X)$. There exists $w \in X$ such that $\mathrm{AM} w=z$. By using the same argument as above, one can easily verify that $\mathrm{S} w=z=A M w$. Now, since the pair (BD, T) and (AM, S) are weakly compatible, then they commute at their coincidence point that is,

$A M z=A M(S w)=S(A M w)=S z$ and $B D z=B D(T u)=T(B D u)=T z$.

Now, we claim that $T z=z$. Let $T z \neq z$. From(2), putting $x=w$ and $y=z$, we have

$$
\begin{array}{r}
d(S w, T z) \lesssim \alpha\left[d(B D z, T z) \frac{1+d(A M w, S w)}{1+d(A M w, B D z)}\right]+\beta[\max \{d(A M w, B D z), d(A M w, S w), d(B D z, T z)\}] \\
+\gamma[d(T z, S w)]+\eta\left[\frac{d(T z, B D z) d(A M w, S w)}{d(T z, A M w)+d(S w, B D z)+d(T z, S w)}\right]
\end{array}
$$

$d(z, T z) \lesssim \alpha\left[d(T z, T z) \frac{1+d(z, z)}{1+d(z, T z)}\right]+\beta[\max \{d(z, T z), d(z, z), d(T z, T z)\}]+\gamma[d(T z, z)]$

$$
+\eta\left[\frac{d(T z, T z) d(z, z)}{d(T z, z)+d(z, T z)+d(T z, z)}\right]
$$

Thus,

$$
d(z, T z) \lesssim \beta d(z, T z)+\gamma d(z, T z)
$$

That is,

$$
|d(z, T z)| \leq(\beta+\gamma)|d(z, T z)|
$$

Which is a contradiction to $(\beta+\gamma)<1$. Therefore $T z=z$. Since $B D z=T z$, which implies that $B D z=z$. Again using (2), putting $x=y=z$, then similarly we can prove that $S z=z$. Since $z=S z$, which implies that $A M z=z=S z$. Now to prove $M z=z$, using (2) putting $x=M z$ and $y=z$, we have

$$
\begin{array}{r}
d(S(M z), T z) \lesssim \alpha\left[d(B D z, T z) \frac{1+d(A M(M z), S(M z))}{1+d(A M(M z), B D z)}\right] \\
+\beta[\max \{d(A M(M z), B D z), d(A M(M z), S(M z)), d(B D z, T z)\}]+\gamma[d(T z, S(M z))]+ \\
d(M z, z) \lesssim \alpha\left[d(z, z) \frac{1+d(M z, M z)}{1+d(M z, z)}\right]+\beta[\max \{d(M z, z), d(M z, M z), d(z, z)\}]+\gamma[d(z, M z)] \\
+\eta\left[\frac{d(T, z) d(M z, M z)}{d(T z, A M(M z))+d(S(M z), B D z)+d(T z, S(M z))}\right]
\end{array}
$$

$d(M z, z) \precsim \beta d(M z, z)+\gamma d(M z, z)$

That is, $\quad|d(z, M z)| \leq(\beta+\gamma)|d(z, M z)|$

Which is a contradiction to $(\beta+\gamma)<1$. Therefore $M z=z$. Since $A M z=z$ which implies that $A z=z$.

Now, to prove $D z=z$, using(2), putting $x=z, y=D z$, we have

$$
\begin{aligned}
& d(S z, T(D z)) \lesssim \alpha\left[d(B D(D z), T(D z)) \frac{1+d(A M z, S z)}{1+d(A M z, B D(D z))}\right] \\
& +\beta[\max \{d(A M z, B D(D z)), d(A M z, S z), d(B D(D z), T(D z))\}]+\gamma[d(T(D z), S z)] \\
& +\eta\left[\frac{d(T(D z), B D(D z)) d(A M z, S z)}{d(T(D z), A M z)+d(S z, B D(D z))+d(T(D z), S z)}\right]
\end{aligned}
$$$$
+\eta\left[\frac{d(D z, D z) d(z, z)}{d(D z, z)+d(z, D z)+d(D z, z)}\right]
$$

$d(D z, z) \lesssim \beta d(D z, z)+\gamma d(D z, z)$

That is, $\quad|d(z, D z)| \leq(\beta+\gamma)|d(z, D z)|$

Which is a contradiction to $(\beta+\gamma)<1$. Therefore $D z=z$. Since $B D z=z$ which implies that $B z=z$. Hence $u$ is a common fixed point of A, B, D, M, S and T.

Uniqueness: From theorem 3.1, we can easily prove the uniqueness of the theorem. Hence A, B, D, M, S and T have a unique common fixed point in $\mathrm{X}$.

\section{Fixed Point Theorem For Weakly Compatible Mapping With CLR Property}

Theorem 3.3 : Let A, B, D, M, S and T be six self mappings of a complex valued metric space (X, d) satisfying:

1.For each $\mathrm{x}, \mathrm{y} \in \mathrm{X}$, there exists $\alpha, \beta, \gamma$ and $\eta$ are non negative real number with $\alpha+\beta+\gamma+\eta<1$, such that $d(S x, T y) \lesssim \alpha\left[d(B D y, T y) \frac{1+d(A M x, S x)}{1+d(A M x, B D y)}\right]+\beta[\max \{d(A M x, B D y), d(A M x, S x), d(B D y, T y)\}]$ 


$$
+\gamma[d(T y, S x)]+\eta\left[\frac{d(T y, B D y) d(A M x, S x)}{d(T y, A M x)+d(S x, B D y)+d(T y, S x)}\right]
$$

2. The pair (AM, S) and (BD, T) are weakly compatible.

3. $\mathrm{S}(\mathrm{X}) \subset \mathrm{BD}(\mathrm{X})$ and the pair $(\mathrm{AM}, \mathrm{S})$ satisfying $\mathrm{CLR}_{\mathrm{AM}}$ property.

4. $\mathrm{T}(\mathrm{X}) \subset \mathrm{AM}(\mathrm{X})$ and the pair $(\mathrm{BD}, \mathrm{T})$ satisfy the $\mathrm{CLR}_{\mathrm{BD}}$ property.

6. The pair (AM, S) and (BD, T) are commute.

Then A, B, D, M, S and T have a unique common fixed point.

Proof: Without loss of generality, assume that $S(X) \subset B D(X)$ and the the pair (AM, S) satisfying CLR $_{\mathrm{AM}}$ property. Then there exists a sequence $\left\{x_{n}\right\}$ in $\mathrm{X}$ such that $\lim _{n \rightarrow \infty} A M x_{n}=\lim _{n \rightarrow \infty} S x_{n}=A M x$ for some $\mathrm{x} \in \mathrm{X}$. Since $S(X) \subset B D(X)$, there exists a sequence $\left\{y_{n}\right\}$ in X such that $S x_{n}=B D y_{n}$. Hence $\lim _{n \rightarrow \infty} B D y_{n}=A M x$. We shall show that $\lim _{n \rightarrow \infty} T y_{n}=A M x$. Let $\lim _{n \rightarrow \infty} T y_{n}=z \neq A M x$. From (1), putting $x=x_{n}$ and $y=y_{n}$, we have

$$
\begin{aligned}
d\left(S x_{n}, T y_{n}\right) \lesssim \alpha\left[d\left(B D y_{n}, T y_{n}\right)\right. & \left.\frac{1+d\left(A M x_{n}, S x_{n}\right)}{1+d\left(A M x_{n}, B D y_{n}\right)}\right] \\
+\beta\left[\operatorname { m a x } \left\{d\left(A M x_{n}, B D y_{n}\right),\right.\right. & \left.\left.d\left(A M x_{n}, S x_{n}\right), d\left(B D y_{n}, T y_{n}\right)\right\}\right]+\gamma\left[d\left(T y_{n}, S x_{n}\right)\right] \\
+\eta\left[\frac{d\left(T y_{n}, B D y_{n}\right) d\left(A M x_{n}, S x_{n}\right)}{d\left(T y_{n}, A M x_{n}\right)+d\left(S x_{n}, B D y_{n}\right)+d\left(T y_{n}, S x_{n}\right)}\right] &
\end{aligned}
$$

Letting $\mathrm{n} \rightarrow \infty$, we have

$d(A M x, z) \lessgtr \alpha\left[d(A M x, z) \frac{1+d(A M x, A M x)}{1+d(A M x, z)}\right]+\beta[\max \{d(A M x, A M x), d(A M x, A M x), d(A M x, z)\}]$

$$
+\gamma[d(z, A M x)]+\eta\left[\frac{d(z, A M x) d(A M x, z)}{d(z, A M x)+d(A M x, A M x)+d(z, A M x)}\right]
$$

$d(A M x, z) \precsim \alpha[d(A M x, z)]+\beta[d(A M x, z)]+\gamma[d(z, A M x)]$

That is, $\quad|d(A M x, z)| \leq(\alpha+\beta+\gamma)|d(A M x, z)|$

Which is a contradiction to $(\alpha+\beta+\gamma)<1$. Therefore $A M x=z$, that is $\lim _{n \rightarrow \infty} T y_{n}=A M x$. Subsequently, we have

$$
\lim _{n \rightarrow \infty} T y_{n}=\lim _{n \rightarrow \infty} S x_{n}=\lim _{n \rightarrow \infty} A M x_{n}=\lim _{n \rightarrow \infty} B D y_{n}=z=A M x
$$

Now, we shall show that $S x=z$. From (1), putting $y=y_{n}$, we have

$$
\begin{aligned}
d\left(S x, T y_{n}\right) \lesssim \alpha\left[d\left(B D y_{n}, T y_{n}\right) \frac{1+d(A M x, S x)}{1+d\left(A M x, B D y_{n}\right)}\right] & \\
+\beta\left[\operatorname { m a x } \left\{d\left(A M x, B D y_{n}\right),\right.\right. & \left.\left.d(A M x, S x), d\left(B D y_{n}, T y_{n}\right)\right\}\right]+\gamma\left[d\left(T y_{n}, S x\right)\right] \\
+\eta\left[\frac{d\left(T y_{n}, B D y_{n}\right) d(A M x, S x)}{d\left(T y_{n}, A M x\right)+d\left(S x, B D y_{n}\right)+d\left(T y_{n}, S x\right)}\right] &
\end{aligned}
$$

Letting $\mathrm{n} \rightarrow \infty$, we have

$$
\begin{aligned}
d(S x, z) \lesssim \alpha\left[d(z, z) \frac{1+d(z, S x)}{1+d(z, z)}\right]+\beta[\max \{d(z, z), d(z, S x), d(z, z)\}]+\gamma[d(z, S x)]+ \\
\eta\left[\frac{d(z, z) d(S x, z)}{d(z, z)+d(S x, z)+d(z, S x)}\right]
\end{aligned}
$$

$d(S x, z) \lesssim \beta[d(S x, z)]+\gamma[d(z, S x)]$

That is, $\quad|d(S x, z)| \leq(\beta+\gamma)|d(S x, z)|$

Which is a contradiction to $(\beta+\gamma)<1$. Therefore $S x=z=A M x$. Now, since the pair (AM, S) is weakly compatible, then they commute at their coincidence point that is, $A M z=A M(S x)=S(A M x)=S z$. Also since $\mathrm{S}(\mathrm{X}) \subset \mathrm{BD}(\mathrm{X})$ there exists $\mathrm{y} \in \mathrm{X}$ such that $z=S x=B D y$. Now, we claim that $T y=z$. From(1), putting $x=x_{n}$, we have

$$
\begin{aligned}
& d\left(S x_{n}, T y\right) \lesssim \alpha\left[d(B D y, T y) \frac{1+d\left(A M x_{n}, S x_{n}\right)}{1+d\left(A M x_{n}, B D y\right)}\right] \\
& +\beta\left[\max \left\{d\left(A M x_{n}, B D y\right), d\left(A M x_{n}, S x_{n}\right), d(B D y, T y)\right\}\right]+\gamma\left[d\left(T y, S x_{n}\right)\right] \\
& +\eta\left[\frac{d(T y, B D y) d\left(A M x_{n}, S x_{n}\right)}{d\left(T y, A M x_{n}\right)+d\left(S x_{n}, B D y\right)+d\left(T y, S x_{n}\right)}\right]
\end{aligned}
$$

Letting $\mathrm{n} \rightarrow \infty$, we have

$$
\begin{aligned}
d(z, T y) \lesssim \alpha\left[d(z, T y) \frac{1+d(z, z)}{1+d(z, z)}\right]+\beta[\max \{d(z, z), d(z, z), d(z, T y)\}]+ & \gamma[d(T y, z)] \\
& +\eta\left[\frac{d(T y, z) d(z, z)}{d(T y, z)+d(z, z)+d(T y, z)}\right]
\end{aligned}
$$


$d(z, T y) \lesssim \alpha d(z, T y)+\beta[d(z, T y)]+\gamma[d(T y, z)]$

That is, $\quad|d(z, T y)| \leq(\alpha+\beta+\gamma)|d(z, T y)|$

Which is a contradiction to $(\alpha+\beta+\gamma)<1$. Therefore $T y=z=B D y$. Now, since pair (BD, T) is weakly compatible, then they commute at their coincidence point that is $B D z=B D(T y)=T(B D y)=T z$.

Now we prove that $S z=T z$, from(1), putting $x=y=z$, we have

$$
\begin{array}{r}
d(S z, T z) \lesssim \alpha\left[d(B D z, T z) \frac{1+d(A M z, S z)}{1+d(A M z, B D z)}\right]+\beta[\max \{d(A M z, B D z), d(A M z, S z), d(B D z, T z)\}] \\
+\gamma[d(T z, S z)]+\eta\left[\frac{d(T z, B D z) d(A M z, S z)}{d(T z, A M z)+d(S z, B D z)+d(T z, S z)}\right] \\
\begin{aligned}
d(S z, T z) \lesssim \alpha\left[d(T z, T z) \frac{1+d(S z, S z)}{1+d(S z, T z)}\right]+\beta[\max \{d(S z, T z), d(S z, S z), d(T z, T z)\}]+\gamma[d(T z, S z)] \\
+\eta\left[\frac{d(T z, T z) d(S z, S z)}{d(T z, S z)+d(S z, T z)+d(T z, S z)}\right]
\end{aligned}
\end{array}
$$

$d(S z, T z) \lesssim \beta[d(S z, T z)]+\gamma[d(S z, T z)]$

That is, $\quad|d(S z, T z)| \leq(\beta+\gamma)|d(S z, T z)|$

Which is a contradiction to $(\beta+\gamma)<1$. Therefore $S z=T z$ that is $A M z=S z=T z=B D z$.

Now, we prove that $z=T z$. From (1), put $\mathrm{y}=\mathrm{z}$, we have

$$
\begin{aligned}
d(S x, T z) \lesssim \alpha\left[d(B D z, T z) \frac{1+d(A M x, S x)}{1+d(A M x, B D z)}\right]+ & \beta[\max \{d(A M x, B D z), d(A M x, S x), d(B D z, T z)\}] \\
& +\gamma[d(T z, S x)]+\eta\left[\frac{d(T z, B D z) d(A M x, S x)}{\mathrm{d}(\mathrm{Tz}, \mathrm{AMx})+\mathrm{d}(\mathrm{Sx}, \mathrm{BDz})+\mathrm{d}(\mathrm{Tz}, \mathrm{Sx})}\right]
\end{aligned}
$$

$\mathrm{d}(\mathrm{z}, \mathrm{Tz}) \lesssim \alpha\left[\mathrm{d}(\mathrm{Tz}, \mathrm{Tz}) \frac{1+\mathrm{d}(\mathrm{z}, \mathrm{z})}{1+\mathrm{d}(\mathrm{z}, \mathrm{Tz})}\right]+\beta[\max \{\mathrm{d}(\mathrm{z}, \mathrm{Tz}), \mathrm{d}(\mathrm{z}, \mathrm{z}), \mathrm{d}(\mathrm{Tz}, \mathrm{Tz})\}]+\gamma[\mathrm{d}(\mathrm{Tz}, \mathrm{z})]$

$$
+\eta\left[\frac{d(T z, T z) d(z, z)}{d(T z, z)+d(z, T z)+d(T z, z)}\right]
$$

Thus, $\quad \mathrm{d}(\mathrm{z}, \mathrm{Tz}) \lesssim \beta \mathrm{d}(\mathrm{z}, \mathrm{Tz})+\gamma \mathrm{d}(\mathrm{z}, \mathrm{Tz})$

That is, $\quad|\mathrm{d}(\mathrm{z}, \mathrm{Tz})| \leq(\beta+\gamma)|\mathrm{d}(\mathrm{z}, \mathrm{Tz})|$

Which is a contradiction to $(\beta+\gamma)<1$. Therefore $\mathrm{Tz}=\mathrm{Sz}=\mathrm{AMz}=\mathrm{BDz}=\mathrm{z}$. similarly by above theorem we can prove that $\mathrm{Mz}=\mathrm{Az}=\mathrm{z}=\mathrm{Dz}=\mathrm{Bz}$.

Uniqueness : From theorem 3.1, we can easily prove the uniqueness of the theorem. Hence A, B, D, M, S and T have a unique common fixed point in $\mathrm{X}$.

\section{Conclusion}

In this paper, we have presented common fixed point theorems in complex valued metric spaces through concept of weak compatibility, E.A. property and CLR property.

\section{Acknowledgments}

The authors thank an anonymous referee for his valuable suggestions that helped to improve the final version of this paper.

\section{References}

[1] A. Azam, B. Fisher, M. Khan, Common fixed point theorems in complex valued metric spaces, Numer. Funct. Anal. Optim. 32 (2011), 243-253, doi: 10.1080/01630 563 .2011.533046.

[2] C. Vetro, On Branciaris theorem for weakly compatible mappings, Appl. Math. Lett., 23 (2010), 700-705, doi: 10.1016/j.aml.2010.02.011.

[3] G. Jungck, Common fixed points for non-continuous non-self mappings on non-metric spaces, Far East J. Math. Sci., 4 (1996), 199212 .

[4] M. Aamri, D.El. Moutawakil, Some new common fixed point theorems under strict contractive conditions, J. Math. Anal. Appl., 270 (2002), 181- 188, doi: 10.1016/S0022-247X(02)00059-8.

[5] R.K. Verma, H.K. Pathak, Common fixed point theorems using property (E.A) in complex valued metric spaces, Thai J. Math., 11 (2013), 347-355.

[6] S. Bhatt, S, Chaukiyal, R.C. Dimri, A common fixed point theorem for weakly compatible maps in complex-valued metric spaces, Int. J. Math. Sci. Appl., 1 (2011), 1385-1389.

[7] S.M. Kang, S. Kumar, M. Kumar and P. Kumar, Common fixed point theorems for weakly compatible mappings in complex valued metric spaces International Journal of Pure and Applied Mathematics Volume 92 No. 3 2014, 403-419

[8] W. Sintunavarat, P. Kumam, Common fixed point theorems for a pair of weakly compatible mappings in fuzzy metric spaces, J. Appl. Math., 2011 (2011), Article ID 637958, 14 pages, doi: 10.1155/2011/637958. 